\title{
OPTIMAL CONTROL OF A SWIRL STABILIZED SPRAY COMBUSTOR USING SYSTEM IDENTIFICATION APPROACH
}

\author{
S.Murugappan, S.Park, A.M.Annaswamy, A.F.Ghoniem \\ Department of Mechanical Engineering, Massachusetts Institute of Technology \\ S.Acharya and D.C.Allgood \\ Department of Mechanical Engineering, Louisiana State University
}

\begin{abstract}
$\underline{\text { Abstract }}$
An optimal controller for a $30 \mathrm{~kW}$ swirl stabilized spray combustor using a system-identification (SI) based model is developed. The combustor was operated in two different configurations. The first consisted of a dual-feed nozzle whose primary fuel stream was utilized to sustain combustion, and the secondary stream was used for active control. The second used a single-feed nozzle with two different swirling air streams. An LQG-LTR (Linear Quadratic Gaussian-Loop Transfer Recovery) controller was designed using the SI based model to determine the active control input, which was in turn used to modulate the secondary fuel stream. Using this controller, the thermoacoustic oscillations, which occurred under lean operating conditions, were reduced to the background noise level. A simpler time-delay controller was also implemented for comparison purposes. The results showed that the LQG-LTR controller yielded an additional pressure reduction of $14 \mathrm{db}$ compared to the time-delay controller in both configurations. This improvement can be attributed to the added degrees of freedom of the LQG-LTR controller that allow an optimal shaping of the gain and phase of the controlled combustor over a range of frequencies surrounding the unstable mode. This leads to the observed further reduction of the pressure amplitude at the unstable frequency while avoiding generation of secondary peaks.
\end{abstract}




\section{Introduction}

Combustion instabilities arise due to positive coupling between acoustic pressure waves and unsteady heat release. These instabilities are often observed in lean premixed gas turbine combustors, ramjet engines, afterburners etc. Pressure oscillations can become significant, leading to violent oscillations in the flow and mechanical vibrations of the system components. Passive control techniques have been used to suppress combustion oscillations. These involve modification to the fuel injection and distribution pattern or the combustor geometry [1]. In recent years, active control has received increasing attention because of its potential as a retrofit technology, and its adaptability over a wide range of operating conditions. Most active control designs use a simple time-

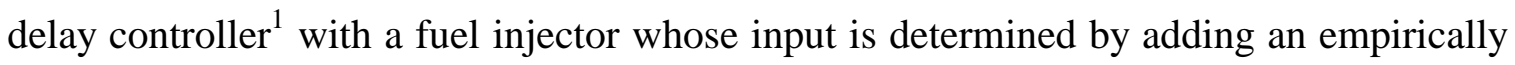
chosen time delay to a filtered pressure signal [2-6]. An alternate approach is to develop model-based active control designs where one can either employ the underlying physics ( e.g., [7-10]), or input-output data together with SI methods (e.g., [11-19]) to derive the model. In this paper, we use the latter approach where suitable inputs and the corresponding pressure outputs are chosen to capture the dominant combustor dynamics.

The system identification method can be viewed as a black-box approach where data from the system is used to fit a particular system model structure, the choice of which is dependent on the main system characteristics that need to be captured. One of

\footnotetext{
${ }^{1}$ The term time-delay controller is used in this paper to describe the commonly used "phase-shift" controller, since the requisite action of delivering the required phase at the unstable frequency is accomplished through the implementation of a time delay to the sensor signal.
} 
the most important features of the pressure/heat-flux sensor signal during unstable combustion is the presence of nonlinear limit cycle oscillations. Following an initial growth in the pressure or heat-flux response, a limit cycle is established due to the effect of system nonlinearities. In [11-14], nonlinear model structures are employed to derive the SI model. In [11], the authors use a nonlinear feedback model where the forward loop contains the linear acoustics, and the feedback loop includes a convective time delay and a nonlinear heat release model. The parameters of these blocks are then identified separately using appropriate input-output data sets. In [12-14], a nonlinear model of the form

$$
\ddot{\eta}+a \dot{\eta}+b \eta=f(\eta, \dot{\eta})+g(\xi(t))
$$

is used, where $a, b$, and $f$ correspond to the self-sustained oscillations in the combustor and $g(\xi(t))$ denotes the effect of an exogenous random noise $\xi$. In [14], $f$ is chosen to be a polynomial function, and data from an experimental rig is used to identify the parameters $a$ and $b$ and the polynomial coefficients. Burgs method [15] and a least-squares method [16] are used to carry out the parameter identification in [13] and [14] respectively.

An alternate approach can be used to model combustion oscillations. Even though the combustion response is nonlinear, in an experimental run, one seldom captures the signal growth within the linear range and transition phase due to its brevity. It is the periodic pressure/heatflux signal, which is the more persistent feature and the one that is experimentally recorded. If it is the periodic oscillations that need to be modeled, one can choose a linear model structure to capture the pressure characteristics. The approach in [17-21] as well as in this paper belongs to this category, where the SI model 
is linear. The implication of such an approach is that in a neighborhood of the limit-cycle oscillations, the linear SI model can accurately predict the combustor response and therefore can be used to design a controller that reduces the amplitude of these oscillations. In [17-20] as well as in this paper, a linear dynamic input-output model structure is chosen as the SI model, whereas in [21], a Fourier-series expansion is used to represent the pressure response. Once the model structure has been selected, several identification methods can be used to determine the model parameters. In [17-20], and in this paper, since the parameters appear as linear coefficients of a differential equation, least squares methods are employed to estimate the parameters [16]. In [21], a nonlinear observer is used to identify all of the parameters in the Fourier series expansion.

The distinction between [17-20] and this paper lies in the process of the validation of the SI model. In [17,18], a laminar $1 \mathrm{~kW}$ combustor is used as an experimental test bed for model validation whereas in [19], simulation studies were carried out using a solid-rocket model in the form of equation (1). In this paper, as well as in [20], a turbulent combustor is the experimental platform for validating SI model-based controllers. In contrast to the $H_{\infty}$ controllers used in [17,18], an LQG-LTR controller is used in this paper since it is more accommodative of saturation constraints in the actuator [22]. Lean operating conditions, where the combustor exhibited strong pressure oscillations, were chosen to test the closed loop control approach. It was observed that the resulting LQG-LTR controller yielded a 12-14 dB higher attenuation of the pressure oscillation over what can be achieved using a time- delay controller as in [2]. The details 
of the experimental setup, the control design, and implementation are discussed in the following sections.

It should be pointed out that the linear model has been derived under the following assumptions: i) the limit cycle response can be approximated by the homogeneous response of a linear differential equation; ii) the effect of an external input on the pressure response can be represented by a linear term. Since (a) the nonlinear response in combustion systems is a stable limit cycle, (b) the sustained pressure response, in most cases, is sinusoidal in nature, and (c) since the effect of a small external input, in general, is small compared to the unforced response, both assumptions( i) and ii) ) are reasonable for pressure responses that are close to the limit cycle. This implies that any model-based controller is guaranteed to be accurate in a neighborhood of the limit cycle, and therefore can reduce its size. The extent of this neighborhood depends on how well the gain and phase of the linear model can approximate that of the nonlinear system as the size of the limit cycle reduces. For all amplitude levels of the pressure where this approximation error remains small, the model-based controller will continue to reduce the amplitude further until the pressure amplitude reaches background noise levels. As will be shown in the experimental studies of the swirl-stabilized combustor at LSU, this was indeed the case and as a result, the model-based LQG-LTR controller reduced the pressure amplitudes all the way down to the noise level.

The advantage of the approach proposed in this paper lies in its generality and simplicity. No information regarding the underlying nonlinearity, instability mechanism, acoustic modes, or any coupling dynamics is necessary to carry out the proposed 
modeling procedure. In contrast, the methods proposed in [11-14] are limited in their scope. As mentioned earlier, the approach proposed therein models combustion dynamics as self-sustained oscillations generated by nonlinear feedback. As is required in most system identification approach that include nonlinear components, system identification is carried out in these papers by assuming a specific form for the nonlinearities, which are either in the form of a polynomial or a saturation. As a result, the fidelity of each nonlinear model thereby derived is determined by the underlying nonlinearities that are actually present in a given combustor. Since there are several distinct nonlinear mechanisms that can all produce a limit-cycle, an approach that is exclusively based on input-output data cannot distinguish between one form of nonlinearity and another, and is therefore limited to a specific configuration.

\section{Experimental Setup}

The experiments were performed in a swirl-stabilized combustor operating at 30 $\mathrm{kW}$ heat release. Two different combustor configurations were used for active control, schematic of which are shown in figure $1 \mathrm{a}$ and $1 \mathrm{~b}$. In both the configurations the air stream entered the combustion chamber at standard temperature, $298 \mathrm{~K}$, and pressure, $1.01 \times 10^{5} \mathrm{~Pa}$. Ethanol was used as a liquid fuel. It was pressurized to an absolute pressure

of $8.27 \times 10^{5} \mathrm{~Pa}$ in a fuel tank using high-pressure inert nitrogen, metered, and supplied to a nozzle through a tube mounted in the center of the air chamber. In the first configuration, which used a dual feed nozzle, air stream with a swirl number equal to 0.8 was used to atomize the fuel. The primary fuel flow rate was kept constant at $2.02 \mathrm{gm} / \mathrm{sec}$ and the average secondary fuel flow rate was set to $0.26 \mathrm{gm} / \mathrm{sec}$ under all operating 
conditions. The airflow rate was varied between 0.014 and $0.035 \mathrm{~m}^{3} / \mathrm{sec}$. The second configuration utilized a single feed Parker Hannifin Research Simplex Atomizer (RSA) nozzle. There were two independent air streams. Primary air stream had a flow rate $0.056-0.283 \mathrm{~m}^{3} / \mathrm{sec}$ which was used for fuel atomization. Secondary air was introduced co-axially around the nozzle with a flow rate that varied from $0.283-1.7 \mathrm{~m}^{3} / \mathrm{sec}$. The average fuel flow rate was kept constant at $0.6 \mathrm{gm} / \mathrm{sec}$. In both the configurations fuel stream could be modulated using an automotive fuel injector driven by a signal processor over a bandwidth of 0 to $400 \mathrm{~Hz}$. The combustion shell was $0.6 \mathrm{~m}$ in length and $0.14 \mathrm{~m}$ in diameter. A high sensitivity, water-cooled pressure transducer was mounted at a normalized axial distance $\mathrm{z} / \mathrm{D}=1.45$, where $\mathrm{z}$ is measured from the nozzle base, as shown in Figure 1a and b, to measure pressure oscillation. Light emissions recorded at the 430nm $\mathrm{CH}$ wavelength using a photodiode was taken as a measure of the heat flux fluctuations from the flame. These signals were then processed in real time using a digital signal processor (DS1103, DSPACE, $333 \mathrm{MHz}$ Motorola power PC) to be used in active control. In order to investigate the combustor dynamics, pressure and photodiode measurements were taken at different equivalence ratios. The entire combustor operating envelope was mapped out as a function of the equivalence ratio, whose value was based on the main fuel stream. A single peak at $205 \mathrm{~Hz}$ was observed, in both the configurations over the entire operating range. Figure 2 shows a typical pressure spectrum at the unstable condition for the first configuration. The frequency of the largest amplitude oscillation corresponded to the quarter wave mode of the combustor [2]. The amplitude of this peak varied depending on the Equivalence ratio. Both pressure and heatflux fluctuations Figure 2: Baseline power spectra for $\phi=0.7$ were normalized by the 
corresponding maximum rms fluctuations and was used as measure of the instability. These are shown in Figure 3. The recorded rms fluctuation of p' varied from 0.2 to 2.7 millibar over an equivalence ratio of 0.6 to 1.5 . The figure illustrates that both the pressure and heatflux oscillations are high near the lean blow out limit. In the first configuration an equivalence ratio of 0.7 , was chosen for the closed loop control study which corresponds to peak instability where $\mathrm{p}_{\mathrm{rms}}=2.7$ millibar. In the second configuration three operating conditions which corresponded to an equivalence ratio of $0.5,0.547$ and 0.74 were chosen for control study.

\section{System Identification of a Combustion System}

System-identification modeling consists of using the input-output data and a black-box approach to derive the model structure and parameters. A typical system identification procedure includes (i) model-structure selection; (ii) determination of the 'best' model in the structure as guided by the data; and (iii) selection of an appropriate excitation signal that includes a wide range of frequencies in order to accurately estimate the model parameters [16]. As mentioned earlier, a typical pressure response in a combustor consists of an initial period where the signal consists of diverging oscillations that are followed by sustained oscillations. We focus on the latter part of the pressure response and choose a linear input-output dynamic model to describe these oscillations. 
This model combines the acoustics, heat release, fuel injector and solid state relay into a single lumped transfer function, which is directly used to design the controller.

With the model-structure selected as a linear dynamic model, we then proceed to part (ii) of the SI procedure, which consists of finding the most accurate linear model given the combustor input-output data. In the current system, the input for system identification is a voltage to the fuel injector, and the output is the pressure signal. The general form of a linear discrete input-output model is given by

$$
y(t)=\sum_{i=1}^{n_{a}} a_{i} y(t-i \Delta t)+\sum_{i=n_{k}}^{n_{k}+n_{b}} b_{i} u(t-i \Delta t)+\sum_{i=0}^{n_{c}} c_{i} e(t-i \Delta t)
$$

where $u(t)$ is the voltage to the injector, $y(t)$ is the pressure signal, $e(t)$ is white noise, $\Delta t$ is the sampling time, $n_{a}, n_{b}, n_{c}$ and $n_{k}$ represent the number of poles, zeros, order of noise and delay in the combustor respectively, and $a_{i}, b_{i}$ and $c_{i}$ are the model parameters. We employ a two-level iteration in order to determine these quantities. The first level of iteration is in the parameter space $\theta$, where

$$
\theta=\left[a_{1}, a_{2} \ldots . a_{n_{a}}, b_{1}, b_{2}, \ldots ., b_{n_{b}}, c_{1}, c_{2} . . c_{n_{c}}\right]
$$

for a given dimension $D=\left[n_{a}, n_{b}, n_{c}, n_{k}\right]$, and the second is in the dimension space. At each iteration, the parameters are adjusted so that a suitable error that reflects the model accuracy is minimized. The details of the two-level iteration are summarized below.

Since the model structure described in equation (2) can be used to capture the periodic nature of the pressure response, our starting point is a model whose output is a 
weighted sum of the past inputs, outputs and the noise. We first select a certain value for $D$. Denoting $\hat{y}(t \mid \theta)$ as the model output, we choose a model as

$$
\hat{y}(t \mid \theta)=\theta^{T} \varphi(t)
$$

where $\varphi(t)$ is a regression vector that is a combination of the past inputs, outputs and noise and is given by

$$
\begin{aligned}
\varphi(t)= & {\left[-y(t-\Delta t), \ldots, y\left(t-n_{a} \Delta t\right),\right.} \\
& u\left(t-n_{k} \Delta t\right), \ldots, u\left(t-\left(n_{k}+n_{b}-1\right) \Delta t\right) \\
& \left., e(t-\Delta t) \ldots e\left(t-n_{c} \Delta t\right)\right]^{T}
\end{aligned}
$$

The goal is to find the optimal value of $\theta$ so that $\hat{y}(t \mid \theta)$ predicts the pressure $y$ as accurately as possible. To achieve this, we construct the error, $\varepsilon(t, \theta)$, defined as

$$
\varepsilon(t, \theta)=y(t)-\hat{y}(t \mid \theta)
$$

and a normalized value of the error $\bar{E}(\theta)$ given by

$$
\bar{E}(\theta)=\frac{1}{N}\left(\frac{\sum_{t=1}^{N}(\varepsilon(t, \theta))^{2}}{(y(t))^{2}}\right)
$$

where $\mathrm{N}$ is the total number of samples. The SI model is then obtained by minimizing $E(\theta)$ over $\theta$. That is, $\theta^{*}{ }_{D}=\underset{\theta}{\arg \min }(\bar{E}(\theta))$

and the resulting $\bar{E}$ is denoted as 


$$
\overline{-}_{D}^{*}=\bar{E}\left(\theta_{D}^{*}\right)
$$

It should be noted here that in order to carry out the minimization in (6), sufficient number of frequencies must be present in the input $u$ so that accurate parameter identification can be carried out. This corresponds to part (iii) of the system-identification procedure. We note that the minimum error $\bar{E}_{D}^{*}$ also varies with $D$. Hence having determined $\theta_{D}^{*}$ and $\bar{E}_{D}^{*}$ for a particular dimension $D$, in the second-level of iteration, we evaluate $\theta_{D}^{*}$ and $\stackrel{-}{E}_{D}^{*}$ for different $D$ to identify the dimension that gives the best SI model. That is, we compute

$$
\overline{-}^{*}=\operatorname{Min}_{D}\left(\bar{E}^{*}\right)
$$

where the best SI model is that which yields $\stackrel{-*}{E}^{*}$.

\section{Implementation}

In order to derive a SI model, a PRBS (Pseudo Random Binary Sequence) signal, low pass filtered at $400 \mathrm{~Hz}$, was chosen to drive the fuel injector so that sufficient number of frequencies are present in the input. The resulting pressure response was recorded using a pressure transducer, and the corresponding power spectrum is shown in Figure 4 for the first configuration. The figure clearly shows a dominant mode at $205 \mathrm{~Hz}$, the same mode captured in the unforced case. There are two other distinct peaks around $60 \mathrm{~Hz}$ and $10 \mathrm{~Hz}$. The $60 \mathrm{~Hz}$ mode is due to the inherent electric noise, while the $10 \mathrm{~Hz}$ mode is associated to the injector dynamics. The latter was confirmed by velocity 
measurements recorded at the exit of the injector for an input white noise. The velocity measurements also indicated that the fuel injector has another mode at $300 \mathrm{~Hz}$. Since the goal of the SI modeling was to represent the combustion dynamics, the fuel injector dynamics was ignored by choosing a band pass filter with a lower and an upper cut-off frequency of 100 and $300 \mathrm{~Hz}$, respectively. The filtered pressure signal was chosen as the output $y$ that had to be modeled. The SI model was then chosen based on the discussion in the previous section.

It was found that the optimal model corresponded to $D=[3,1,1,0], \theta_{D}^{*}=[-2.44$, $\left.2.32,-0.82 ; 4.6 \times 10^{-5}\right], \quad \bar{E}(\theta)=12.5 \%$ for the dual-feed nozzle. The structure of $D$ indicates that a third order model was sufficient to predict the combustor dynamics. This is also corroborated in Figure 5, which shows the power spectrum of the SI model predicting the peak at $205 \mathrm{~Hz}$.

The same procedure was utilized for the single-feed nozzle to develop models at three different operating conditions that correspond to equivalence ratios of $\phi=0.5$, $\phi=0.547$ and $\phi=0.74$. Table 1 shows the SI results for the single-feed nozzle case.

\section{$\underline{\text { LQG-LTR Control }}$}

For a high order unstable system, a classical time-delay controller [23,24] is inadequate to stabilize the system because it lacks requisite degree of freedom in gain and phase. One way to overcome this deficiency is to use the LQG-LTR method [25]. This method provides sufficient performance and robustness over a wide range of frequencies [22]. An LQG-LTR controller has the form: 


$$
u=-\left[K(s I-A-B K-H C)^{-1} H\right] y
$$

where the matrices $A, B$ and $C$ are obtained from the combustor state-space model, and the estimator gain, $H$, and the state feedback gain, $K$, are to be designed. The feedback gain, $K$, is determined using the performance index $J$ given by

$J=\int_{0}^{\infty}\left(y^{T} Q y+u^{T} R u\right) d t$

$Q=I, R=\rho I$

where $\rho$ is a scaling factor that determines the trade-off between fast transients and the magnitude of the control input. $H$ can be found in a similar way as $K$ by posing the problem as the design of a Kalman filter where one introduces input noise with a variance $I$ and output noise with a variance $\mu I$ where $\mu$ represents the model uncertainty. $H$ and $K$ can then be found by fine tuning $\rho$ and $\mu$ using the MATLAB control system toolbox.

\section{Controller Design and Implementation}

The discrete time combustor for the dual-feed nozzle obtained previously is cast in continuous-time using Tustin's method [26]. The resulting expression is

$$
W(s)=\frac{-7.010 * 10^{-6}(s-4000)(s+4000)^{2}}{\left(s^{2}+2 \varsigma w_{n} . s+w_{n}{ }^{2}\right)(s+364.7)}
$$


where $\varsigma=0.0185$ and $w_{n}=1287 \mathrm{radian} / \mathrm{sec}$. Using this model, an LQG-LTR controller was designed using MATLAB. The control parameters $\rho$ and $\mu$, were varied to obtain the maximum attenuation in pressure oscillation. The controller has the form:

$$
W_{c}(s)=\frac{-0.4963 s^{2}-1004 s-2.53 \cdot 10^{5}}{s^{3}+732.1 s^{2}+1.9^{*} 10^{6} s+7.6 \cdot 10^{8}}
$$

with $\rho=1$ and $\mu=1 \mathrm{e}-6$. The same procedure was used for the single-feed nozzle case. The resulting controller transfer functions are made using the combustor model given in Table 1 for all the three operating conditions.

In order to perform real-time control a super scalar microprocessor Motorola power PC 604e running at $333 \mathrm{MHz}$ and a slave DSP TMS320F240 were used. The latter has 16 input channels and 8 output channels with A/D's at 16 bit and D/A at 14 bit with the latter having a $+-10 \mathrm{~V}$ range and a $20 \mathrm{MHz}$ clock rate. Code generation, compiling and downloading was done with SIMULINK and DSPACE real time interface. A sampling time of $0.1 \mathrm{msec}$ was chosen to implement the control algorithms. The output of the D/A board was then fed to a solid-state relay to run the automotive fuel injector on the secondary stream.

\section{$\underline{\text { Results }}$}

\section{Dual-feed Nozzle Case}

An operating condition corresponding to an equivalence ratio of 0.7 and p'rms $=2.7 \mathrm{mbar}$ was chosen to implement the active controllers. The LQG-LTR controller 
resulted in pressure and heat release responses whose spectra are shown in Figure 6a and 6b. These figures also show the power spectra of the uncontrolled (baseline) system. The performance of the LQG-LTR controller is also compared with the more commonly used time-delay controller [2]. The latter consisted of a filter-time delay-amplifier combination, where the filter attenuated frequencies outside the band [150, $350 \mathrm{~Hz}]$. The time delay, $\tau_{p s}$, was varied between 0 and $4.8 \mathrm{msec}$, and the amplifier gain was fixed at 100. The gain was chosen so as to reduce the pressure to the levels shown in figure. The choice of the time delay was on an empirical basis. The impact of the time delay on the pressure amplitude is shown in Figure 7. As can be seen in Figure 7, a maximum

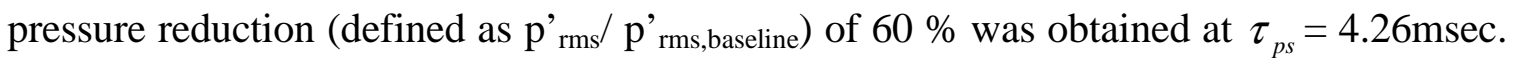
In contrast, the maximum pressure reduction with the LQG-LTR was $80 \%$. Three different measures of performance were chosen to compare the LQG-LTR controller with the phase-shift controller: (i) maximum pressure reduction ratio in frequency domain, $R_{\omega}$, given by

$$
R_{\omega}=\frac{\hat{P}_{m L Q G-L T R}}{\hat{P}_{m \text { Phasedelay }}}
$$

where $\hat{P_{m}}=\underset{w \in[100,300] \|}{\operatorname{Max}}\|\hat{P}(w)\|$ and $\hat{P}$ denotes the power spectrum of the pressure response, (ii) a time-averaged energy norm, $R_{t}$, of the pressure which is given by

$$
R_{t}=\frac{\left.\int\left(p^{\prime}\right)^{2} d t\right|_{\text {phase }}-\left.\int\left(p^{\prime}\right)^{2} d t\right|_{L q g}}{\left.\int\left(p^{\prime}\right)^{2} d t\right|_{\text {phase }}} \cdot 100 \%
$$


and (iii) a filtered energy-norm (from $100 \mathrm{~Hz}$ to $300 \mathrm{~Hz}$ ), $R_{t f}$, of the pressure. It was found that $R_{\omega}=0.22$. For measure (ii) and (iii), $R_{t}$ and $R_{t f}$ are computed and it was found that $R_{t}=54.7 \%$ and $R_{t f}=40.7 \%$.

\section{Single-feed Nozzle Case}

The combustion dynamics was modeled using the system identification procedure at three different operating conditions of $\phi=0.5, \phi=0.547$, and $\phi=0.74$ as shown in Table 1. SI-based LQG-LTR controllers were designed and implemented for these cases, and in each of these cases, the weighting parameters $\rho$ and $\mu$ were fine-tuned to optimize the performance. The corresponding measures (i) to (iii) were obtained and are shown in Table 2 for the three operating conditions. The phase shift controller was implemented at the same operating conditions. The measures $R_{t}$ show that the effectiveness of the LQG-LTR controller decreases at $\phi=0.547$ and slightly decreases at $\phi=0.5$ compared to the pressure reduction at $\phi=0.74$. The reason for this nonuniform performance may be due to the following: As shown in Figure 8, the pressure amplitude changes drastically around $\phi=0.5$ which is near the lean blow-out limit (LBO) as $\phi$ changes. This sensitivity of the pressure amplitude near the LBO changes the model parameters rapidly. This rapid change of the model parameters can generate a large modeling error if $\phi$ is perturbed by the control input. This increased modeling error may affect the effectiveness of LQG-LTR control at $\phi=0.547$. 


\section{$\underline{\text { Discussion }}$}

The results in the previous section show the improvement achieved when using an LQG-LTR controller, compared to the time-delay controller common to both configurations. In this section, we discuss possible reasons for this improvement. As will be shown, the time-delay controller adds a fixed gain and time delay to the pressure signal, whereas the LQG-LTR controller optimizes the profile of the gain and phase to achieve the desired goal.

By construction, the gain of the time-delay controller is a constant over all frequencies. To increase the effectiveness of the controller at the unstable frequency, this gain must be large. At frequencies where the phase of the forward-loop transfer function of the system together with controller is close to $0^{\circ}$, a large gain can excite the corresponding frequency. Thus, the gain must be kept reasonably low to avoid exciting secondary modes. This limits the effectiveness of the controller. On the other hand, the gain of the LQG-LTR controller reaches a maximum around the unstable frequency. This allows the controller to suppress the dominant oscillation effectively. At the same time, the gain of the LQG-LTR drops rapidly on either sides of unstable frequency. Since secondary peaks are generated at points where the gain of the open-loop transfer function of the system (controller+combustor) is greater than 1 millibar/volt and the phase is near $0^{\circ}$ (positive feedback), and since the LQG-LTR controller has a small gain at all values away from the unstable frequency, the controller prevents the excitation of secondary modes. 
The time-delay controller has a single parameter, which is the value of the time delay, that can be adjusted to affect the slope of the phase, as shown in Figure 9. Even though the added time delay corresponds to a correct phase at the primary mode, it may give the wrong phase at other frequencies. Figure 10 shows the forward-loop transfer function of the controller together with the combustor. The resulting closed-loop system can generate a secondary peak with the time-delay controller because the phase crosses $0^{\circ}$ line at $\omega=185 \mathrm{~Hz}$. At this frequency, any perturbations present can be amplified if the gain is larger than 1 millibar/volt. If the gain at this frequency is reduced to be less than one, the gain plot of the phase-shift*combustor transfer function in Figure 10 indicates that the gain at the unstable frequency is also reduced to a value less than 4.8 . This value, however, may be too small for the time-delay controller to be effective enough to result in pressure suppression. This limitation is not present in the LQG-LTR controller, since as shown in Figure 9, the corresponding phase does not cross $0^{\circ}$ at any frequency.

In summary, two properties of the LQG-LTR controller contribute towards not exciting any secondary peaks. These include: the rapid roll-off of the gain around the unstable frequency, and the small change of the phase away from the unstable frequency so as to avoid cross-over of the $0^{\circ}$ line, both of which are not present in the time-delay controller. Both of these properties are due to the fact that the LQG-LTR controller allows many degrees of freedom in its gain and phase by virtue of the fact that it has several parameters ( twice the order of the controller). This is in contrast to the timedelay controller which has only two parameters, the gain and the delay. 


\section{$\underline{\text { Summary and Concluding Remarks }}$}

In this paper, a system-identification method was used to develop a model for a swirl stabilized spray combustor operating at $30 \mathrm{KW}$. An LQG-LTR controller designed using the SI model reduced the pressure and photodiode oscillations to the background noise level. A simpler time-delay controller was also implemented for comparison purposes and it was observed that the LQG-LTR controller provided 12-14 db higher reduction over the former for both the combustor configurations. Analysis using SI based model showed that the LQG-LTR controller allows many more degrees of freedom than the time delay controller, as a result of which, the LQG-LTR controller effectively suppresses the pressure oscillations by carefully tailoring the gain and phase over the entire spectrum.

The approach adopted in this paper consisted of modeling the pressure response of the combustor near the limit-cycle. The sustained pressure oscillations were modeled as a linear system response, which is guaranteed to approximate the actual nonlinear response of the combustor in a neighborhood of the limit cycle. The fact that the controller, which was based on the linear model, reduced the pressure oscillations down to the background noise indicates that the approximation error in modeling the limit cycle response of the combustor as a linear model is quite small.

The approach suggested in this paper can be adopted to reduce pressure oscillations in any combustor rig. It should be however noted that in general, a single linear model may not be sufficient to reduce pressure oscillations to the background noise 
level. As mentioned earlier, the amount of reduction of pressure oscillations that the controller can achieve is restricted by the size of a domain where a linear model can represent the nonlinear dynamics. Once the system leaves this domain, the linear model may not be accurate, and as a result, the model-based controller may become ineffective. It should however be noted that a repeated application of the same procedure can reduce the pressure oscillations further [20]. That is, once the model-based controller results in a reduced limit-cycle, the resulting closed-loop system can be identified once again with linear model structures. The resulting linear model can in turn be used to design yet another LQG-LTR controller. Using such a family of linear models and model-based LQG-LTR controllers, one can reduce the pressure oscillations systematically. Since this step-by-step process is simple and straightforward, one can generate an algorithm to implement this process automatically. Our current efforts are focused on using such an iterative closed-loop identification and control procedure for pressure suppression in large-scale rigs.

\section{$\underline{\text { Acknowledgements }}$}

This collaborative work between MIT and LSU is supported by the Propulsion Program of the office of Naval Research, grant No. N00014-97-0957 (LSU) and N0001499-1-0448 (MIT), and in part by the National Science Foundation, grant No. ECS 9713415 (MIT). The support of Honeywell Engines is gratefully acknowledged. The authors would also like to thank Ravi Prasanth of Scientific Systems, Inc. for several insightful discussions on system-identification. 


\section{$\underline{\text { References }}$}

[1] K.C. Schadow, E. Gutmark, K.J. Wilson and R.A.Smith, "Multistep Dump Combustor Design to Reduce Combustion Instabilities," Journal of Propulsion and Power, Vol. 6, No. 4. 1990.

[2] S. Murugappan, S. Acharya, E.J. Gutmark and T. Messina. "Characteristics and control of combustion instabilities in a swirl-stabilized spray combustor," AIAA/ASME/SAE/ASEE Joint Propulsion Conference and Exhibit, $35^{\text {th }}$, Los Angeles, CA, June 20-24, 1999.

[3] G.J. Bloxsidge, A.P. Dowling, N. Hooper and P.J. Langhorne. "Active control of an acoustically driven combustion instability," Journal of Theoretical and Applied mechanics, supplement to Vol.6, 1987.

[4] K. Yu, K.J. Wilson and K.C. Shadow. "Scale-Up experiments on liquid-fueled active combustion control," AIAA Paper 98-3211, 1998.

[5] E. Gutmark, T.P. Parr, K.J. Wilson, D.M. Hanson-Parr and K.C. Shadow. "Closedloop control in a flame and a dump combustor," IEEE Control Systems, 13:73-78, April 1993. 
[6] W. Lang, T. Poinsot and S. Candel. "Active control of nonlinear pressure oscillations in combustion chambers," Journal of Propulsion and Power, Vol. 8, No. 6, 1992.

[7] J. Rumsey, M. Fleifil, A.M. Annaswamy, J.P. Hathout and A.F. Ghoniem. "The role of active control in suppressing thermoacoustic instability," Proceedings of the American Control Conference, Albuquerque, New Mexico, June 1997.

[8] V. Yang, A. Sinha, and Y.T. Fung. "State feedback control of longitudinal combustion instabilities," Journal of Propulsion and Power, Vol.8, No.1,1992.

[9] A.M. Annaswamy, M. Fleifil, J.P. Hathout, and A.F. Ghoniem. "Impact of linear coupling on the design of active controllers for thermoacoustic instability," Combustion Science and Technology, 128:131-180, December 1997.

[10] M. Fleifil, J.P. Hathout, A.M. Annaswamy and A.F. Ghoniem. "Reduced order Modeling of heat release dynamics and active control of time-delay instability," AIAA Aerospace Science Meeting Conference and Exhibit, $38^{\text {th }}$, Reno, Nevada, January 10-13, 2000.

[11] R.M. Murray, C.A. Jacobson, R. Casas, A.I. Khibnik, C.R. Johnson, R. Bitmead, A.A. Peracchio, W.M. Proscia. "System Identification for limit cycling systems: A case study for combustion instabilities," American Control Conference, Philadelphia, PA, June 24-26, 1998. 
[12] V.S. Burnley. “ Nonlinear Combustion instabilities and stochastic sources,” PhD. Thesis, California Institute of Technology, 1996.

[13] F.E.C. Culick, L. Paparizos, J. Sterling, V.Burnley. "Combustion Noise and Combustion Instabilities in Propulsion systems," Proceedings of AGARD Conference on combat aircraft noise, AGARD CP512, 1992.

[14] T.C. Lieuwen, D. Lee. “ Nonlinear modeling of combustor dynamics using experimental data," AIAA/ASME/SAE Joint propulsion conference, $36^{\text {th }}$, Huntsville, AL, July 2000.

[15] J.P. Burg. "Maximum Entropy Spectral Analysis," Annual international meeting, $37^{\text {th }}$, Soc. Of Explor. Geophys., Okhlahama city, 1967.

[16] L. Ljung. System Identification: Theory for the User. $2^{\text {nd }}$ edition. Prentice-Hall Inc., Upper Saddle River, N. J., 1999.

[17] J.E. Tierno and J.C. Doyle. "Multimode active stabilization of a Rijke tube," In DSC-Vol. 38. ASME Winter Annual Meeting, 1992. 
[18] R.K. Prasanth, R. Mehra and A.M. Annaswamy. "A system identification model of the MIT laminar Combustor and Model Based Control," Technical Report, Adaptive Control Laboratory, Department of Mechanical Engineering, MIT, 1999.

[19] S. Koshigoe, T. Komatsuzaki and V. Yang. "Adaptive control of combustion instability with on-line system identification," Journal of Propulsion and Power, Vol. 15, No. 3, pp.383-389, May-June 1999.

[20] B.J Brunell. "A System Identification Approach to Active Control of Thermoacoustic Instability,” M.S. Thesis, Department of Mechanical Engineering, MIT, 2000.

[21] Y. Neumeier and B.T. Zinn, “ Active Control of Combustion instabilities with real time observation of unstable combustor modes," AIAA Aerospace Science Meeting Conference and Exhibit, 34 ${ }^{\text {th }}$, Reno, NV, January, 1996.

[22] A.M. Annaswamy, M. Fleifil, J.W. Rumsey, R. Prasanth, J.P. Hathout, and A.F. Ghoniem. "Thermoacoustic Instability: Model-Based Optimal Control Designs and Experimental Validation,” IEEE Transactions on Control systems Technology, 2000.

[23] J.P Hathout, A.M. Annaswamy, M.Fleifil and A.F. Ghoniem. "A Model-Based Active Control Design for Thermoacoustic Instability," Combust. Sci. and Tech., Vol 132, pp.99-138, 1998. 
[24] K. Ogata. Modern Control Engineering, third Edition, Prentice-Hall, Inc., 1997.

[25] G. Stein and M. Athans. "The LQG/LTR procedure for multivariable feedback control design," IEEE Transactions on Automatic Control, 32:105-114, February 1987.

[26] G.F.Franklin, J.D.Powell, M.Workman. Digital Control of Dynamic Systems, Third edition, Addison Wesley, 1997. 

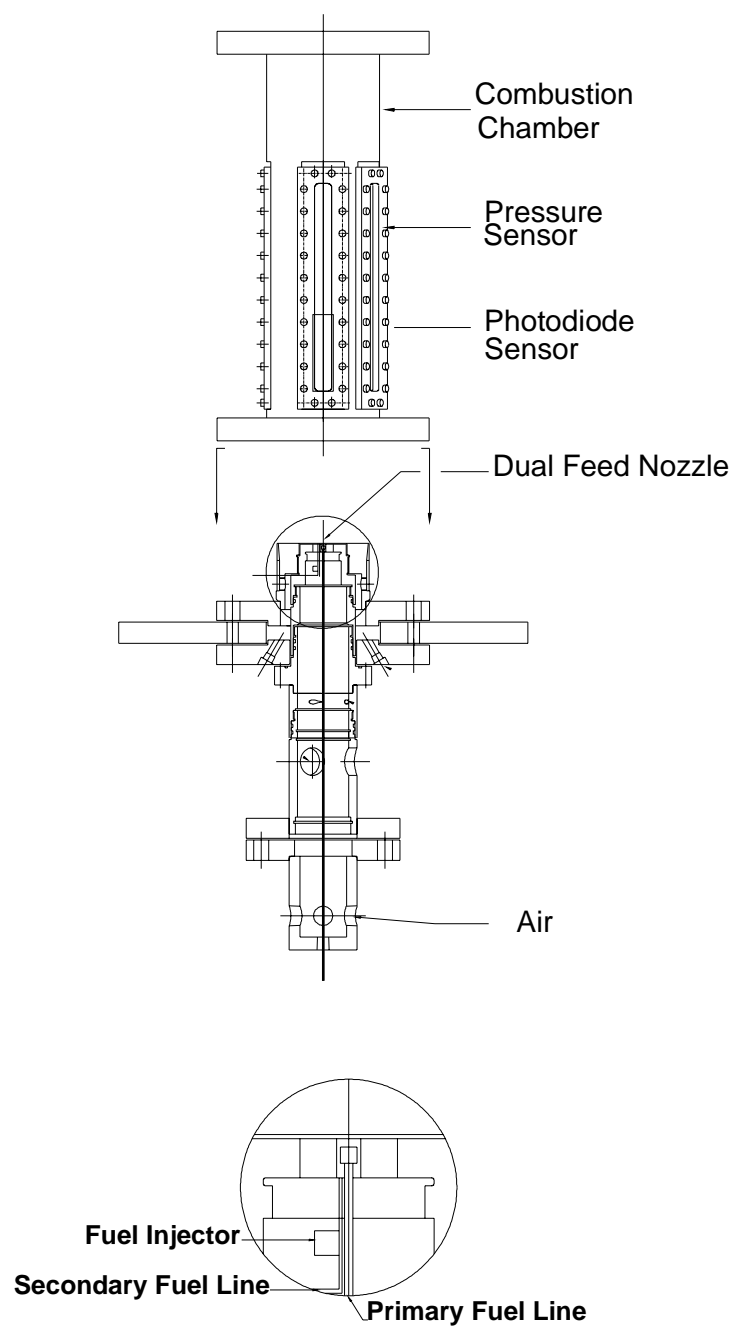

Figure 1a Schematic diagram of combustor (Configuration 1) 


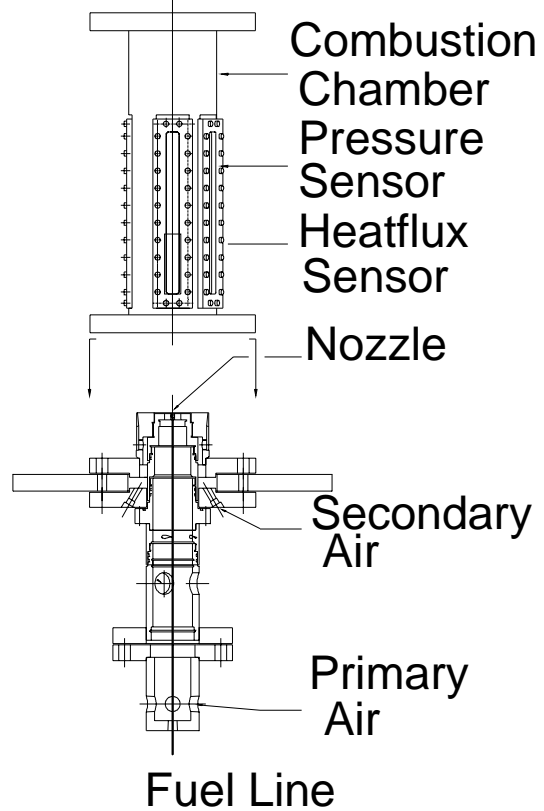

Figure $2 \mathrm{~b}$ Schematic diagram of combustor (Configuration 2) 


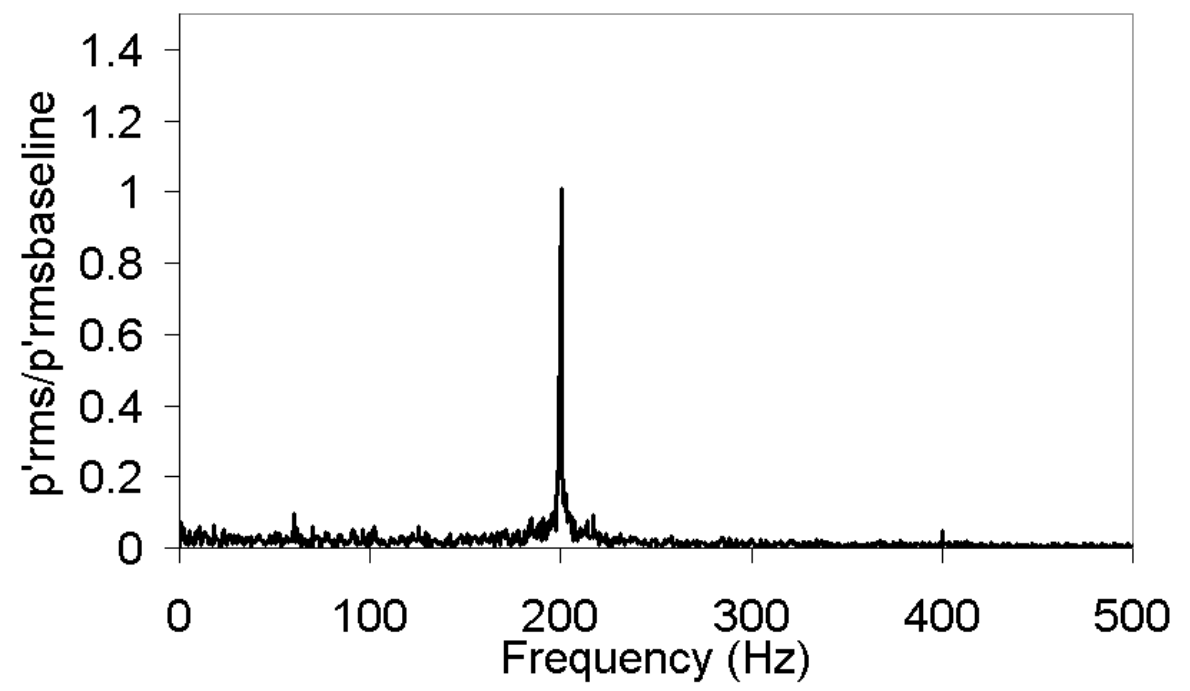

Figure 3 Baseline power spectra for $\phi=0.7$.

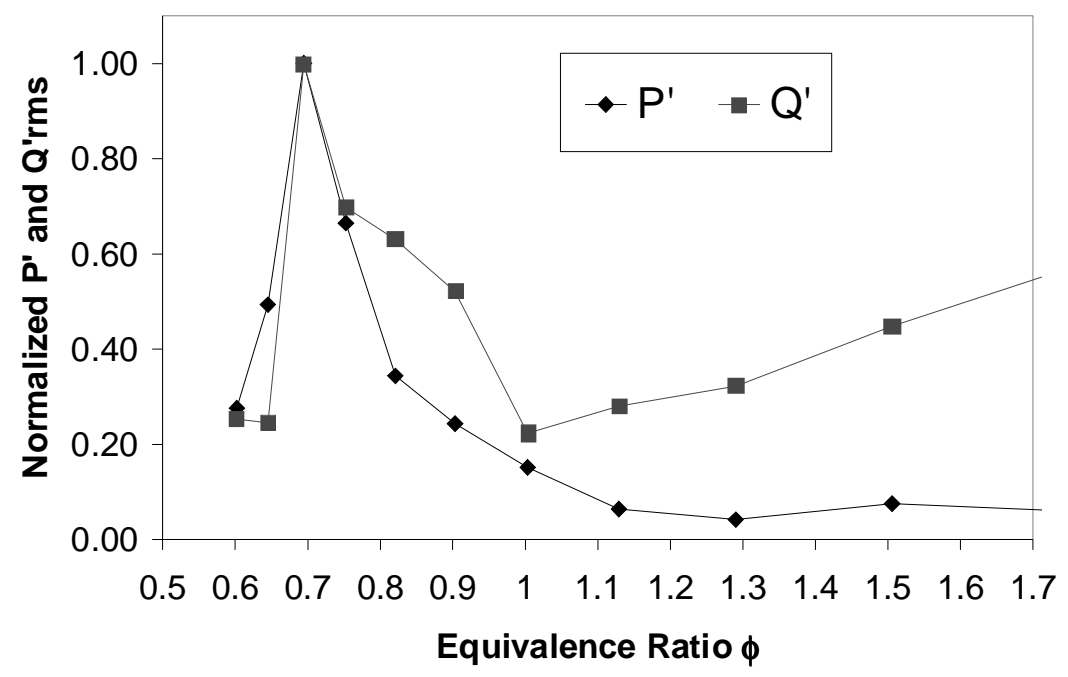

Figure 4 Normalized p'rms and q'rms as functions of primary fuel equivalence ratio for the dual-feed nozzle case 


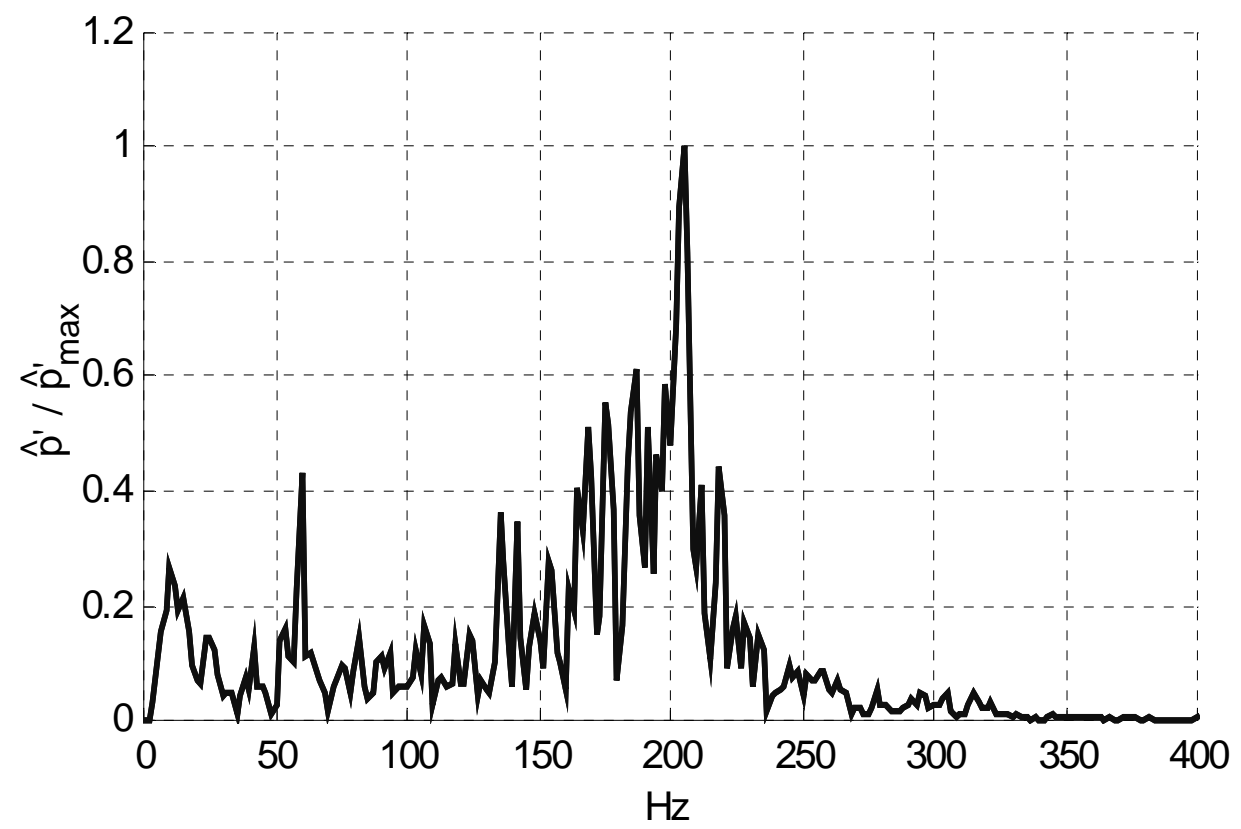

Figure 5 Power spectra of the pressure signal with PRBS input at $\phi=0.7$

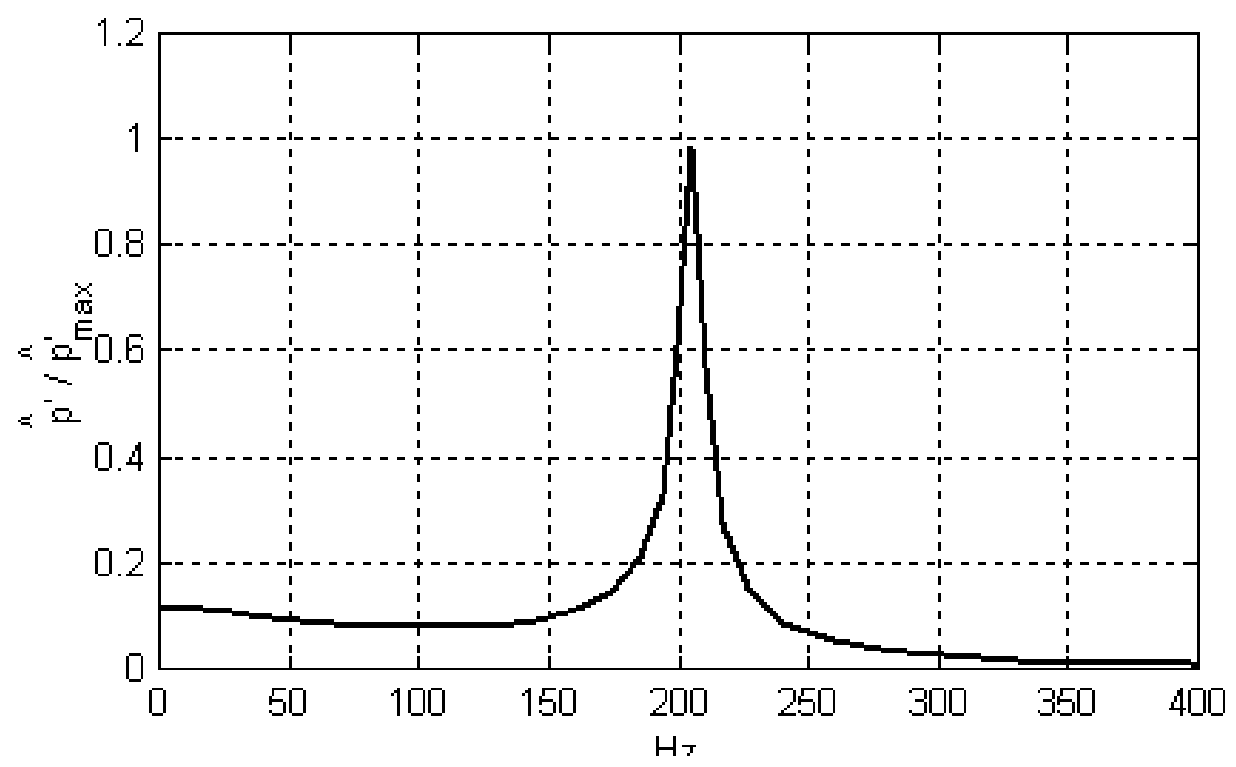

Figure 6 Power spectra of the system-identification model at $\phi=0.7$ 


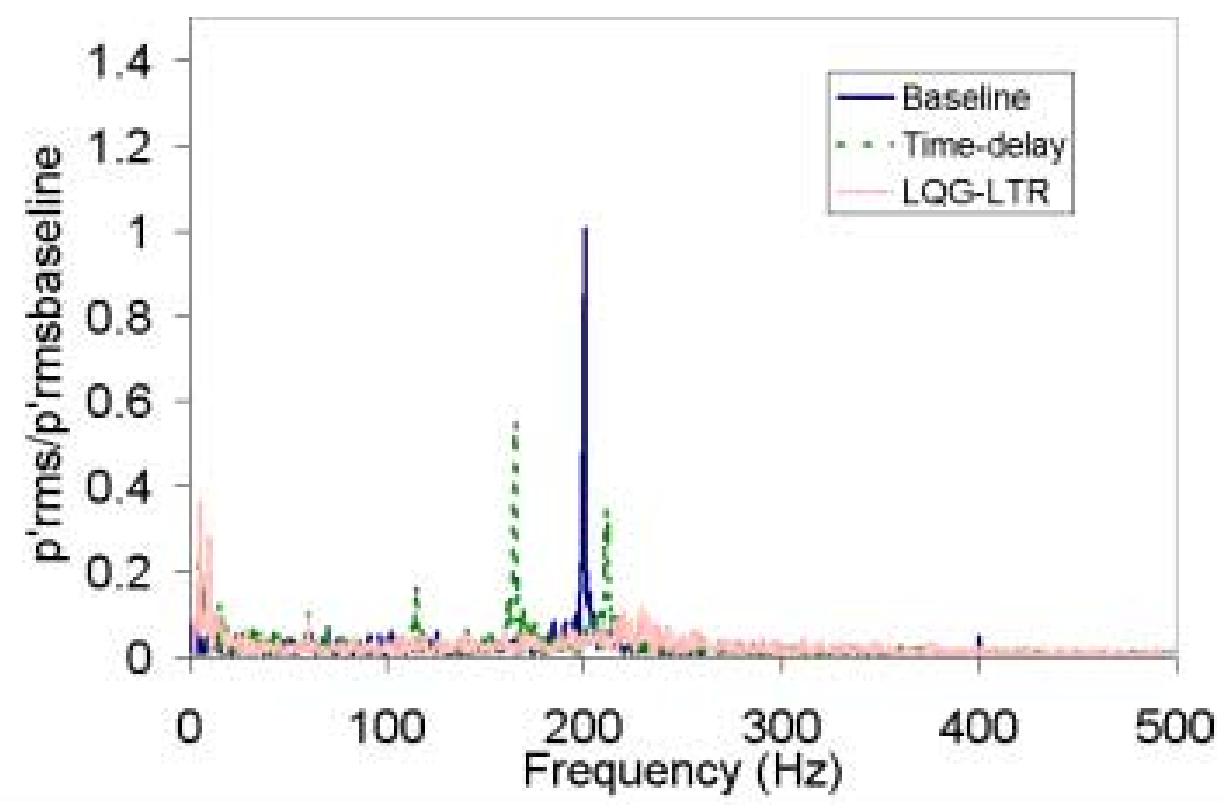

Figure 7a p'rms spectra at the baseline, time- delay and LQG-LTR control at $\phi=0.7$

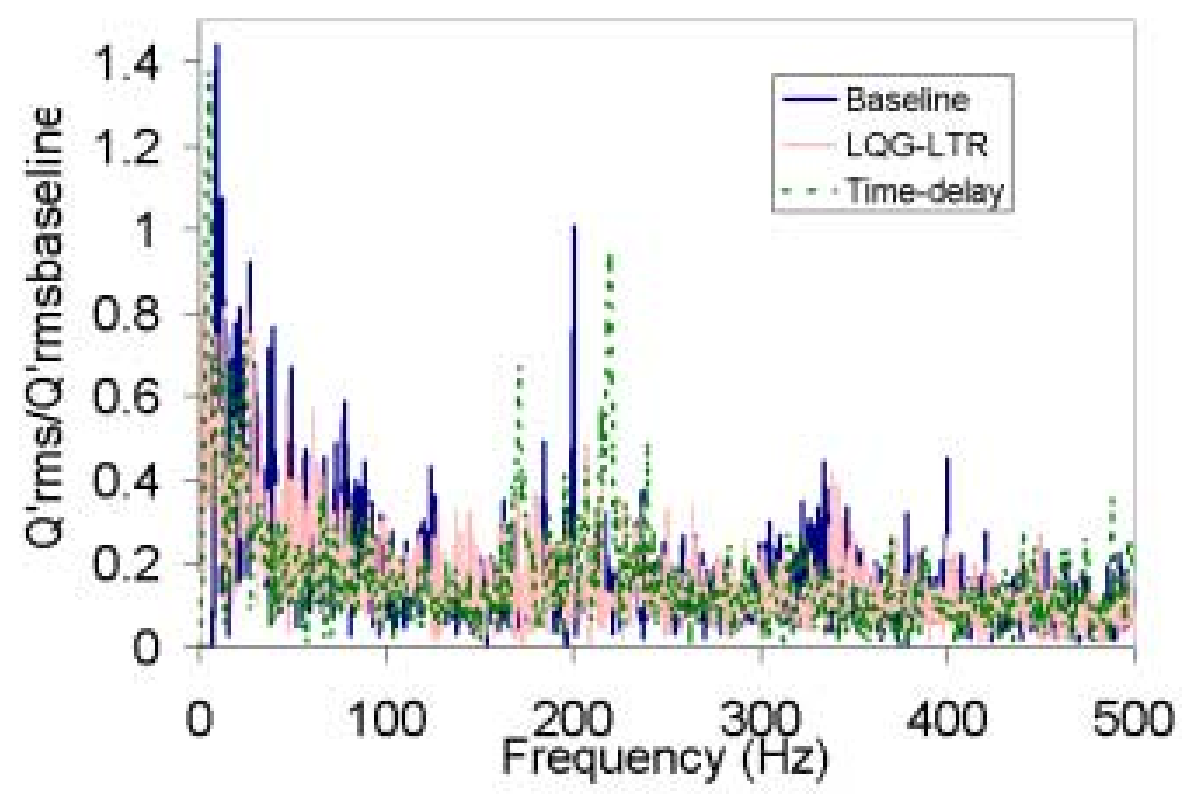

Figure 6b Q'rms spectra at the baseline, time- delay and LQG-LTR control at $\phi=0.7$. 


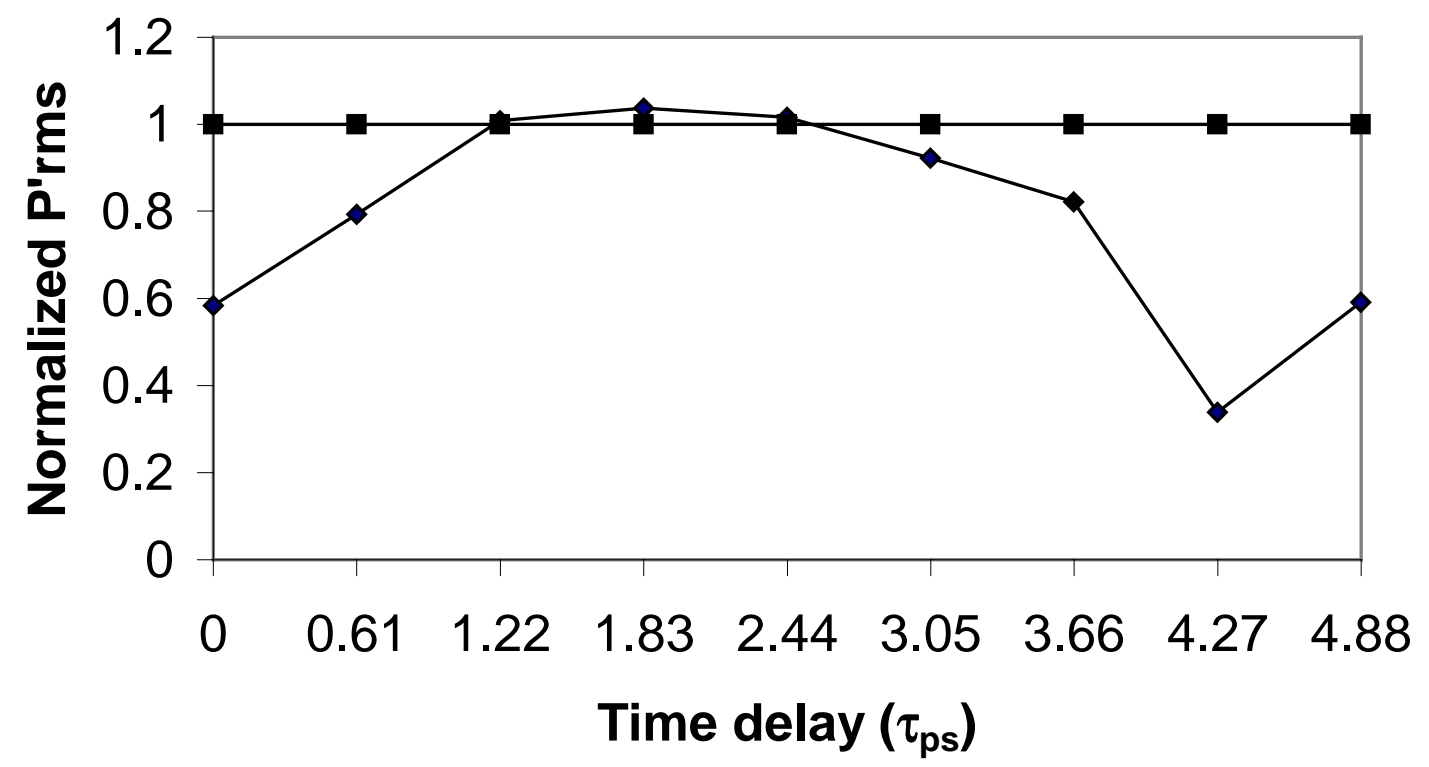

Figure 8 Normalized p'rms for different time delays at $\phi=0.7$.

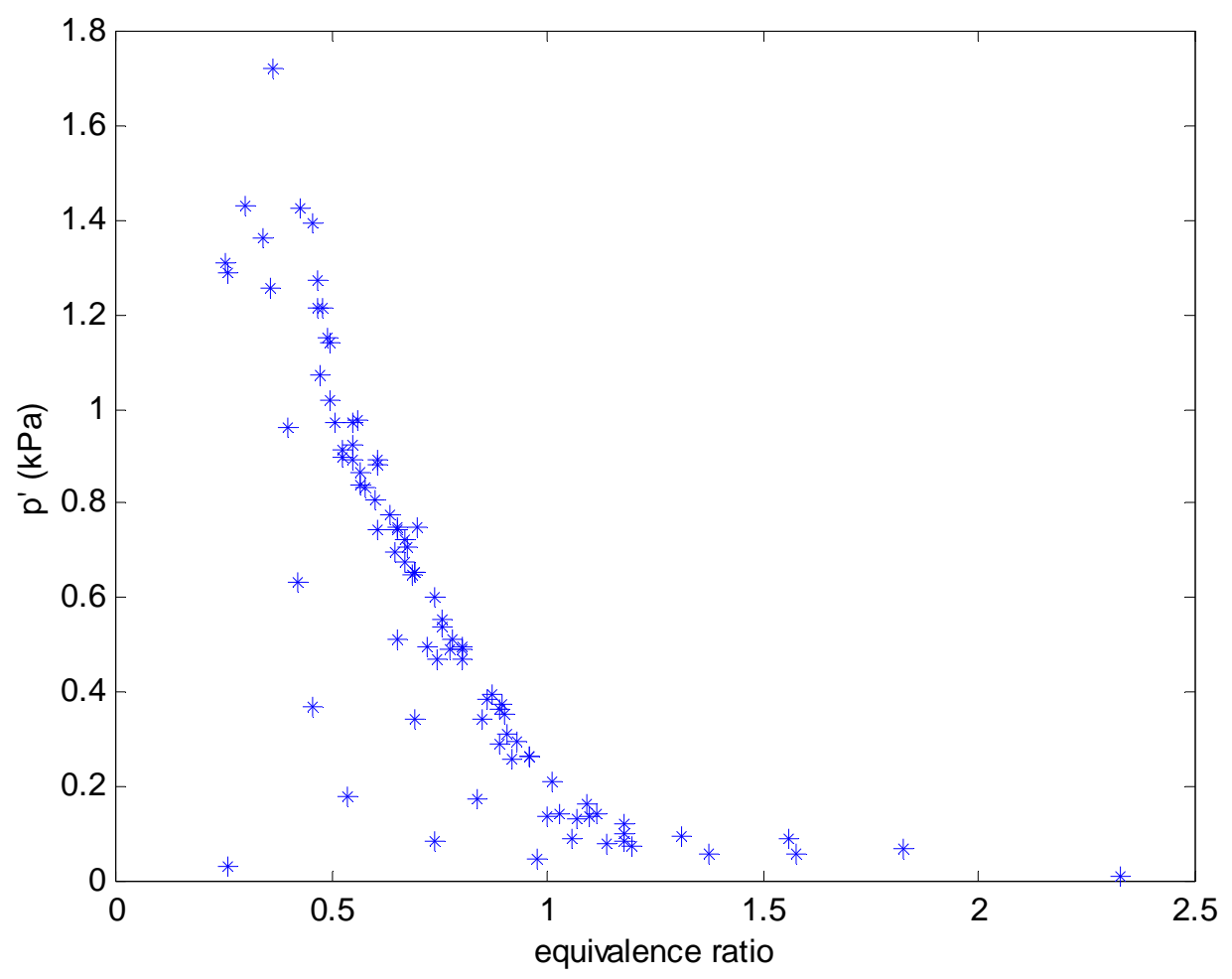

Figure 9 Normalized p'rms as functions of primary fuel equivalence ratio for the singlefeed nozzle case 

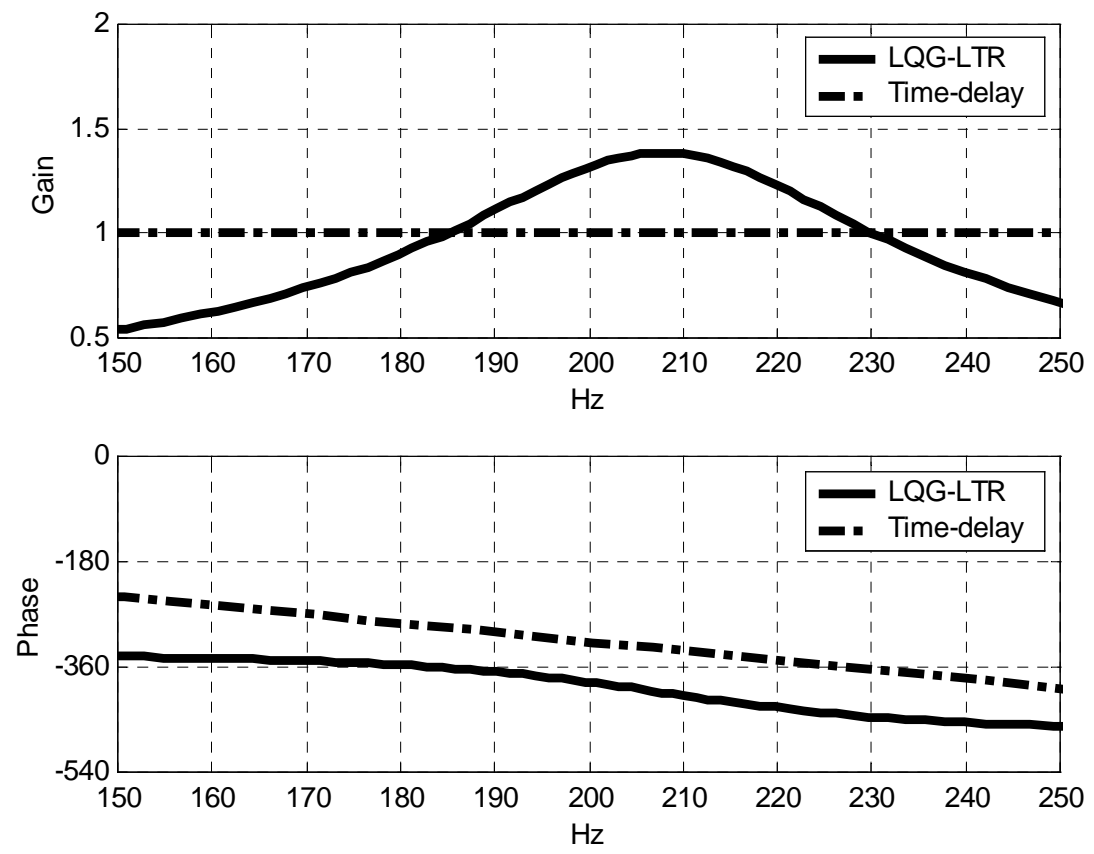

Figure 10 Bode plot of LQG-LTR and the time-delay controller at $\phi=0.7$
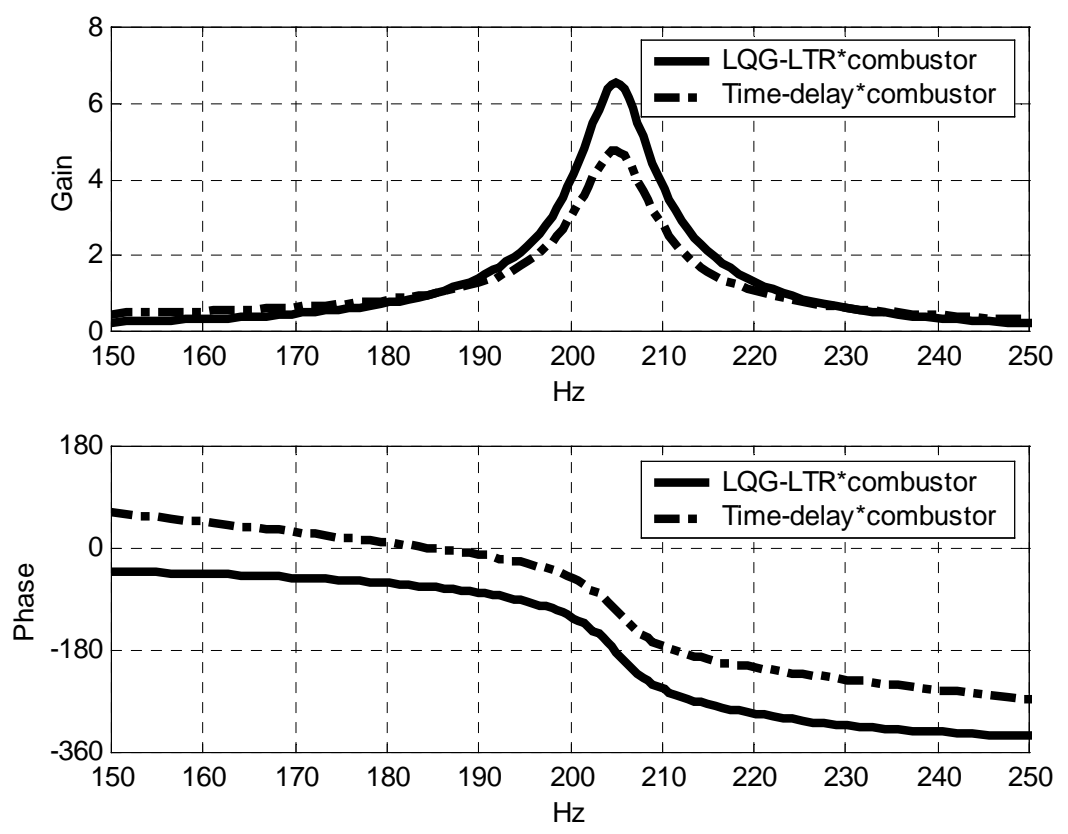

Figure 11 Open-loop transfer functions of the system (combustor*controller) at $\phi=0.7$ 
Table 1 Optimal Models in a single-feed nozzle case at different equivalence ratios

\begin{tabular}{|c|c|c|}
\hline $\begin{array}{c}\text { Equivalence } \\
\text { ratio }\end{array}$ & $\mathrm{D}$ & $\theta_{D}^{*}$ \\
\hline 0.5 & {$[10,10,1,2]$} & $\begin{array}{rrrrr}-7.8017, & 29.1651, & -68.2139, & 110.1133, & -127.9216, \\
108.2487, & -65.9227, & 27.7078, & -7.2862, & 0.9181 ; \\
0.0012, & -0.0105, & 0.0407, & -0.0950, & 0.1477, \\
-0.1595, & 0.1198, & -0.0606, & 0.0188, & -0.0028]\end{array}$ \\
\hline 0.547 & {$[2,2,2,5]$} & {$[-1.6243,0.9982 ;-0.0010,0.0007 ; 0.1706,-0.8143]$} \\
\hline 0.74 & {$[10,10,1,1]$} & $\begin{array}{rrrrr}-7.8790, & 29.7354, & -70.0994, & 113.8331 & -132.7505, \\
112.5253, & -68.5012, & 28.7280, & -7.5271, & 0.9449 ; \\
0.0003, & -0.0015, & 0.0032, & -0.0030, & -0.0009, \\
0.0058, & -0.0074 & 0.0051, & -0.0020, & 0.0003]\end{array}$ \\
\hline
\end{tabular}

Table 2 Reduction rate in the time window

\begin{tabular}{|c|c|c|c|}
\hline & $R_{\omega}$ & $R_{t}$ & $R_{t f}$ \\
\hline Dual-feed nozzle case & 0.22 & $55 \%$ & $41 \%$ \\
\hline Single-feed Nozzle case & & & \\
\hline$\phi=0.5$ & 0.25 & $64 \%$ & $72 \%$ \\
\hline$\phi=0.547$ & 0.20 & $27 \%$ & $51 \%$ \\
\hline$\phi=0.74$ & 0.22 & $80 \%$ & $91 \%$ \\
\hline
\end{tabular}

